University of Redlands

\title{
Integrating BIM with ArcGIS for Indoor Navigation
}

\author{
A Major Individual Project submitted in partial satisfaction of the requirements \\ for the degree of Master of Science in Geographic Information Systems \\ by \\ Mengchao Xu \\ Douglas Flewelling, Ph.D., Committee Chair \\ Fang Ren, Ph.D.
}

June 2015 
Integrating BIM with ArcGIS for Indoor Navigation

Copyright (C) 2014

by

Mengchao Xu 
The report of Mengchao Xu is approved.



Fang Ren, Ph.D.



Nov-2016 



\section{Acknowledgements}

I would like to express my gratitude towards my advisor, Dr. Douglas Flewelling, for his patience and encouragement that help me in completion of this project. My thanks and appreciations also go to Dr. Ihab Hijazi, this project would not have been possible without his support and help. 



\author{
Abstract \\ Integrating BIM with ArcGIS for Indoor Navigation \\ by \\ Mengchao Xu
}

With increasing demand for indoor navigation and rapid developments in Building Information Modeling (BIM), indoor routing and analysis attracts attention from both the GIS and architecture worlds. This project's goal was to integrate BIM with GIS and utilize it for indoor navigation use. It aimed to provide executable methods in ArcGIS for indoor path generation and to explore the possibilities for further applications.

In this project, Data Interoperability Extension was used to operating the transformation from Industry Foundation Classes (IFC) to geodatabase. After importing the data, two methods were proposed: Mesh and TIN. The Mesh method used a standardsized grid graph as the referencing network for a floor and subsequently mapping the movement on a $2 \mathrm{D}$ plane to the movement along grid edges. TIN method utilized the TIN network as the base; it maps the movement on a 2D plane to the movement along TIN edges. Both of the methods were achieved by using tools and functions in ArcGIS. In conclusion, the result shows that the Mesh approach provided a very precise network for the building floor, whereas the TIN approach was efficient on the generating process side. 



\section{Table of Contents}

Chapter 1 - Introduction ......................................................................................... 1

$1.1 \quad$ Client

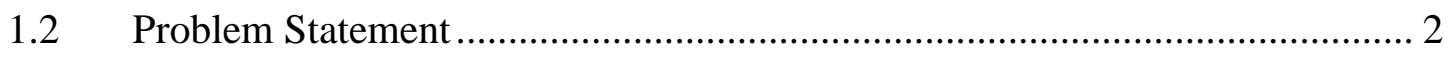

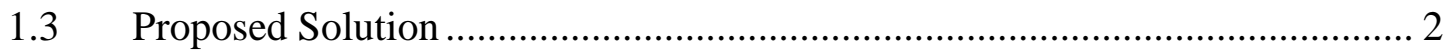



1.3.2 Scope

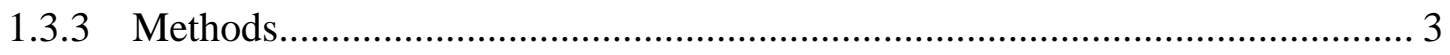



1.5 Overview of the Rest of this Report ............................................................. 4

Chapter 2 - Background and Literature Review ................................................................ 5

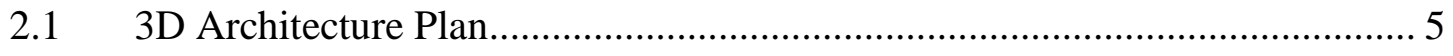

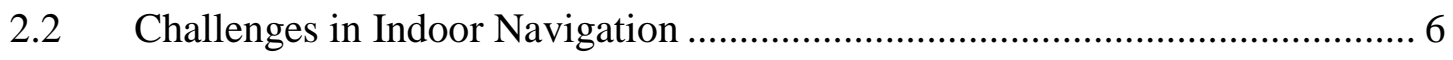

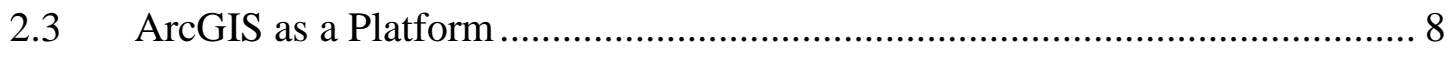

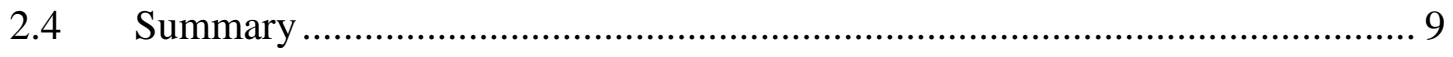

Chapter 3 - Systems Analysis and Design........................................................................... 11

3.1 Problem Statement ............................................................................. 11

3.2 Requirements Analysis .......................................................................... 11

3.2.1 Functional Requirements ...................................................................... 12

3.2.2 Non-Functional Requirements .................................................................. 12

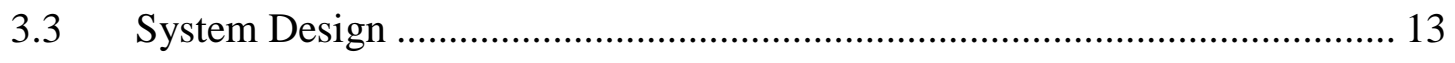

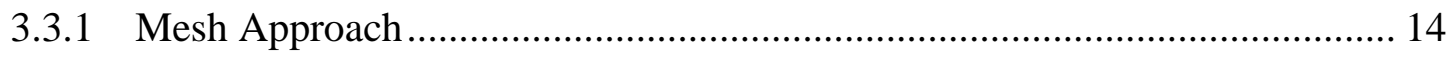

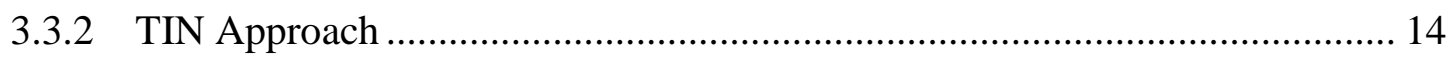


3.3.3 Proof for Completeness.......................................................................... 15

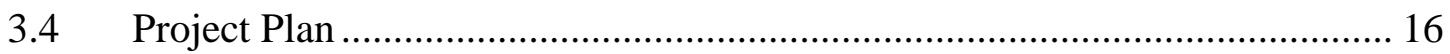

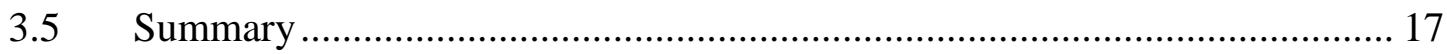

Chapter 4 - Data Import and Preparation ........................................................................ 19

4.1 Data Source and IFC Data Model ............................................................ 19

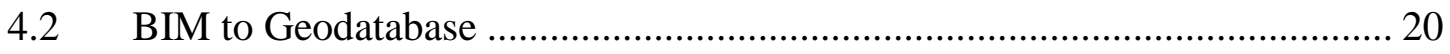

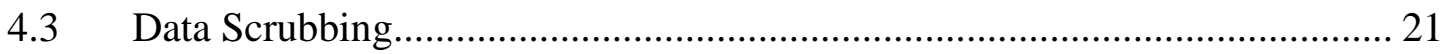

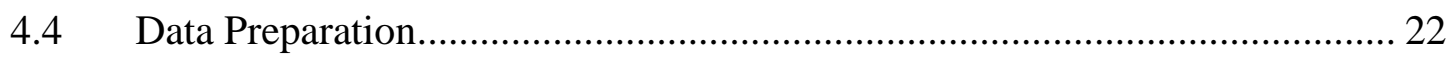



Chapter 5 - Implementation................................................................................................... 25



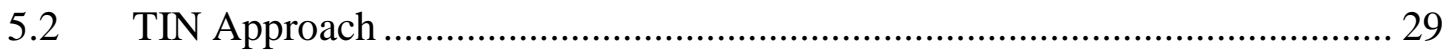

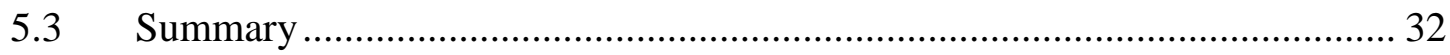

Chapter 6 - Results and Analysis.......................................................................................... 33

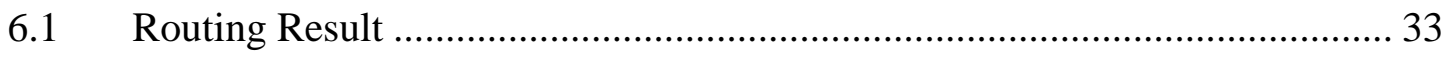

6.2 Comparison between Mesh and TIN Approaches .......................................... 34

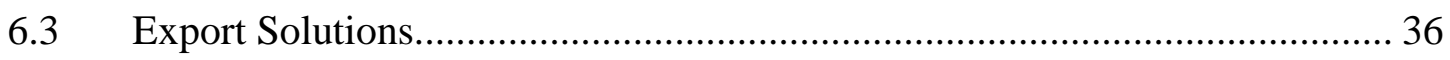



Chapter 7 - Conclusions and Future Work .................................................................. 37

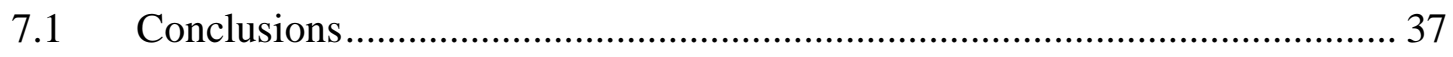

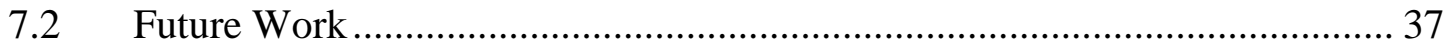

Appendix A. Workflows-Mesh ......................................................................................... 43 
Appendix B. Workflows-TIN............................................................................................ 44

Appendix C. Workflows-Data Preparation................................................................. 45 



\section{Table of Figures}

Figure 2-1: A Building Information Model in IFC Format ............................... 6

Figure 2-2: $\quad$ Reflex Angled Corners …....................................................... 8

Figure 3-1: $\quad$ Project System Design ......................................................... 14



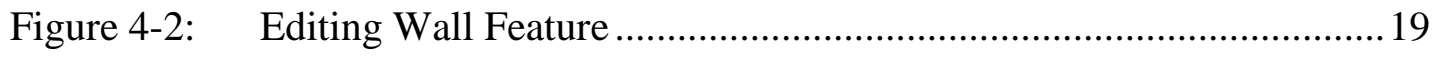



Figure 5-1: $\quad$ Preparing a Floor Plan .............................................................. 24

Figure 5-2: $\quad$ Creating a Fishnet Template for a Floor Plan ..................................2 25

Figure 5-3: $\quad$ Mesh Network for Multiple Floors …...........................................26

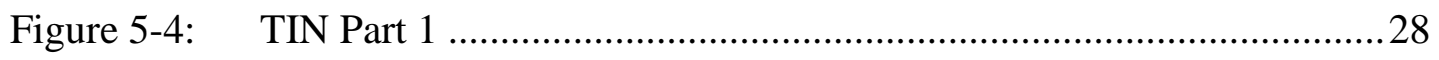

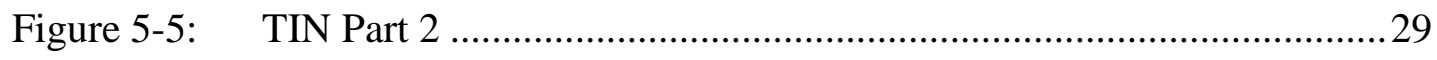

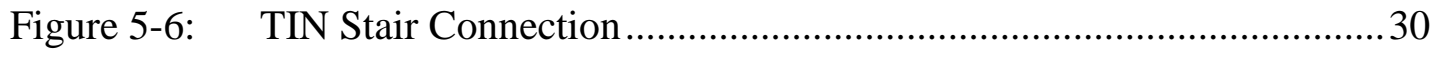

Figure 5-7: $\quad$ TIN Network for Multiple Floors …............................................. 30

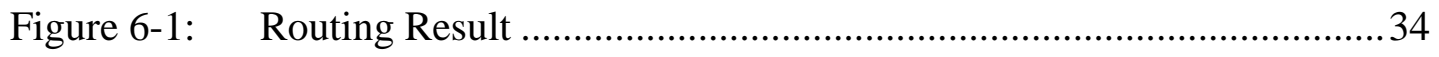

Figure 6-2: $\quad$ Solved Z-Hall Way Problem........................................................ 35 



\section{List of Tables}

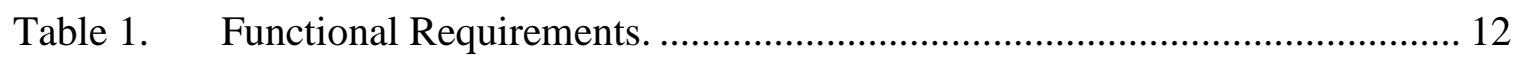

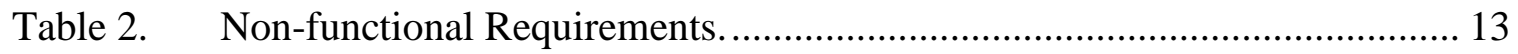

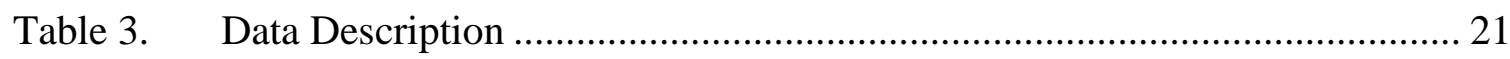

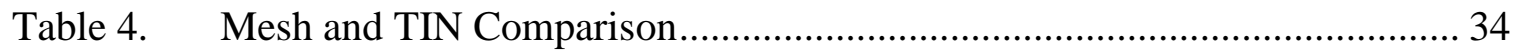





\section{List of Acronyms and Definitions}

$\begin{array}{ll}\text { AEC } & \text { Architectural, Engineering, and Construction } \\ \text { BIM } & \text { Building Information Modeling } \\ \text { BIMs } & \text { Building Information Models } \\ \text { CAD } & \text { Computer-aided design } \\ \text { CDT } & \text { Constrained Delaunay Triangulation } \\ \text { GIS } & \text { Geographic Information System } \\ \text { GSA } & \text { General Services Administration } \\ \text { IAI } & \text { International Alliance for Interoperability } \\ \text { IFC } & \text { Industry Foundation Classes } \\ \text { NBS } & \text { National Building Specification } \\ \text { POI } & \text { Points of Interest } \\ \text { TIN } & \text { Triangulated Irregular Network }\end{array}$





\section{Chapter 1 - Introduction}

Navigation is a traditional topic in geography: one of the most useful applications of maps has always been the tracking of paths from one location to another and storing the routes. Outdoor routing analysis is simpler than it once was with digitized maps and geodatabases. In GIS software like ArcGIS, finding the shortest path between two locations and performing routing analysis are no longer tedious tasks once the network dataset is created using the road layer and points of interest (POI) as inputs.

As one of the fastest-growing GIS areas, indoor GIS is booming because of the rapid development of three-dimensional (3D) technology and increased demands from industries including but not limited to robotics, architecture, transportation, and city management. The applications such as assets detection and indoor dynamic evacuation planning are showing the value of indoor GIS analysis. Research work regarding navigation in indoor environment is one of the fundamental studies in this area. However, considering the complexity of indoor environments, indoor navigation is never an easy job. GIS researchers face various challenges such as lack of complete building data, low accuracy of indoor positioning, and inhomogeneity of path generation. Nevertheless, path generation is the basis for navigation analysis. This report presents a project that integrated Building Information Models (BIMs) with ArcGIS and employed two approaches to generating indoor path networks.

This chapter provides an overview of this project. Section 1.1 introduces the project's client. A detailed statement about the problem is found in Section 1.2. Section 1.3 gives the proposed solutions for the problem. The audience for this project is defined in Section 1.4. Section 1.5 offers a brief synopsis of the rest of this report. 


\subsection{Client}

This project's client was Dr. Ihab Hijazi, a postdoctoral scholar from ETH (Zurich) information architecture group. His has extensive experience in the use and application of computer programs in the field of Architecture and Urban Planning. Dr. Ihab provided data and goals for this project, he's job was to both provide necessary knowledges from architecture field and exam the final output for acceptance.

\subsection{Problem Statement}

There is a demand in both indoor GIS and architecture fields for simple and effective methods of generating path network dataset for architectural plans. In most cases, a path network dataset is required before performing any kind of network analysis. This is not usually a concern for outdoor GIS analysis since road data already carry basic information about the paths. For indoor environments, 3D architecture plans such as BIMs do not have the path feature embedded as a layer. As such, the problem addressed in this project was how to integrate BIM with GIS software to support indoor navigation.

\subsection{Proposed Solution}

The general solution for this project was to develop executable methods or workflows in ArcGIS to generate the indoor path network for 3D architectural floor plans. Methods based on Mesh and Triangulated Irregular Network (TIN) were applied for this project. Mesh approach focused on increase the precision of the path network; TIN was designed to both increase the efficiency of data usage and decrease the difficulty of generating process. 


\subsubsection{Goals and Objectives}

The goal of this paper was to find an automatic and executable way to generate network datasets for BIMs in GIS. This project sought to create effective methods for indoor navigation, utilize BIMs with GIS for the purpose of indoor navigation and to discover path generation methods for BIMs in ArcGIS. By creating methods and workflows in ArcGIS, users would be able to use them as guidelines and adapt them for other buildings.

\subsubsection{Scope}

This project was a research-based study. The idea was to utilize GIS functions in ArcGIS to generate network datasets for BIMs. The scope consisted of a detailed description of the workflow on generating path network datasets for BIMs and an example of applying it. The deliverables are workflows which enables 3D architecture plans for indoor navigation.

\subsubsection{Methods}

The project data came in the form of Industry Foundation Classes (IFC) files provided by the client. After importing them into geodatabases using Data Interoperability in ArcGIS 10.2, the rest of the project was completed using Model Builder and the editing tools in ArcGIS 10.2. ArcScene was the main working environment utilized in this project.

\subsection{Audience}

The audience for this report is GIS and architecture professionals. This report acts a referencing document for professionals interested in using BIMs with ArcGIS for indoor navigation and other purposes. The audience also includes those seeking solutions and 
applications for indoor path generation from 3D building data. The potential applications include evacuation planning, indoor accessibility evaluation, and solving locationallocation problems in indoor environment. Bringing commonly used analyses for outdoor environment and customize them for indoor space is valuable for further investigation.

\subsection{Overview of the Rest of this Report}

Chapter 2 introduces the 3D architecture plan, the Building Information Modeling (BIM) and Industry Foundation Classes (IFC) formats, followed by discussions of previous work done in the indoor path generation field. The system design and plan are addressed in Chapter 3. Chapter 4 describes the necessary data preparation and editing works before operating the methods. Chapter 5 provides detailed descriptions about the project implementation, including step-by-step instructions for each of the two methods. Chapter 6 discusses the final outputs from each method and contains a quality analysis. The conclusion of this project and discussions about the future work can be found in Chapter 7. 


\section{Chapter 2 - Background and Literature Review}

Considering the complexity of indoor environments, indoor navigation faces a significant number of challenges. Some well-established outdoor navigation methods like GPS are not usually available (Nagel et al., 2010). In general, Building Information Models (BIMs) are 3D architecture plan files that can be used to support decision-making about a place. According to Autodesk (Autodesk, 2014), BIMs are 3D, object-oriented and Architectural, Engineering, and Construction (AEC)-specific CAD. This project used BIMs as the data source for path generation.

Section 2.1 gives a brief introduction on BIM and IFC format data, followed by a discussion in Section 2.2 of several existing methods that people offer for indoor path generation. Section 2.3 and 2.4 focus on the possibility of using ArcGIS as a platform to perform indoor navigation. The last section provides a summary for this chapter.

\subsection{D Architecture Plan}

Building Information Modeling is the process of generating and managing 3D building during its life circle; BIM is used to increase the productivity of design, construction, and maintenance. Generally, it is the process of adding intelligence to 3D CAD data and be treated as 3D architecture plans. More importantly, its purpose is to make the design information explicit, so that the design intent and program can be immediately understood (GSA, 2007). Presently, there is an expanding interest in integrating BIM with GIS. BIM systems contain objects with the maximum level of detail in 3D geometry (Irizarry \& Karan, 2012). On the other hand, GIS is strong in 2D geometry and it is starting to experiment with 3D (Berlo \& Laat, 2010). Importing BIMs into geodatabases and working with them has become increasingly essential for spatial analysis. 




\section{Figure 2-1: A Building Information Model in IFC Format}

Industry Foundation Classes (IFC) is a commonly used file format in the construction industry for data interoperability, and BIM data is usually stored as IFC files; it contains the graphic representation of spatial objects and their relationships in a logical view. It is registered as an official International Standard ISO 16739:2013. According to BuildingSMART, formerly the International Alliance for Interoperability (IAI), IFC define data elements that represent the parts of buildings and their spatial relations (El-Mekawy, 2010). It works as a holder of BIM data, and the FME reader gallery in ArcGIS includes it as a resource for interoperability. Figure 2-1 is a Building Information Model in IFC format.

\subsection{Challenges in Indoor Navigation}

Many researchers investigated indoor navigation methods in the past few years. Indoor navigation methods can be divided roughly into two categories: geometrical and graphical. The difference between geometrical and graphical approaches is in the level of accuracy, where the geometrical is more accurate due to utilizing quantitative methods in determining geometric path, while graphical methods use topology of premises for planning of the paths in navigation. The graph-based models were improved through integrating semantics with the model. 
A basic indoor navigation system requires an indoor path network and a positioning method. Graph networks are the basis of routing. They are the topological representations of space, and a path usually consists of a set of linked edges leading from the starting point to the endpoint (Rüppel, Abolghasemzadeh, \& Stübbe, 2010). Generating paths requires abstracting space as nodes and connecting them by edges. If mapping a 3D building into a network topological system is the goal, the main challenges become the partitioning of space and connecting the subdivisions. Depending on the accuracy requirement, the path network could be generated into different levels of detail.

Depending on the method of splitting the space and levels of abstraction employed by the user, a path network could be used as a point-to-point guide, turn-byturn reference or room-to-room guide. One way to generate path network automatically is to use a grid-based graph as the referencing path network. All the rooms and hallways are covered with nodes and, if they are in accessible areas, these nodes are connected with edges (Rüppel, Abolghasemzadeh, \& Stübbe, 2010). Shrinking the cell size and dividing the space into more subdivisions can improve the graph. However, this method has low efficiency in computation and ultimately requires extra storage space.

Constrained Delaunay Triangulation (CDT) is a generalization of the Delaunay triangulation that forces certain required segments into the triangulation (Chew, 1987). For a 2D plane, it could be used to decompose space into triangular cells. In order to generate a path network, the centers of triangles are taken as the nodes and connected adjacently (Xu et al., 2013). This method is a proper solution for regular shaped structure; however, for long and narrow L-shaped hallways, it is hard to use straight lines to connect adjacent points without crossing the corner walls. In extreme 
cases, shown in Figure 2-2, such as Z-shaped hallways with obtuse or even reflex angled corners, using a straight line to connect two adjacent points without hitting the walls is impossible.

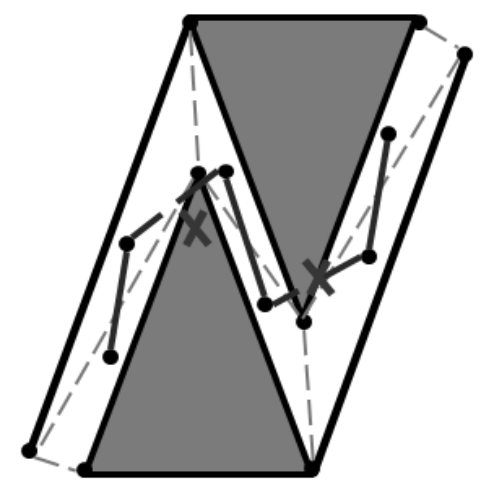

\section{Figure 2-2: Reflex Angled Corners}

Lamarche and Donikian (2004) suggested spatial subdivision to assist real-time navigation in complex indoor environments. This approach uses the minimal distance between corners and walls, together with Delaunay triangulation to split space. It then runs the convex cell optimization process to simplify the subdivisions and minimize the number of cells. Finally, in extracting the topological relations for the space, a node represents a corresponding cell and an edge represents a segment shared by adjacent cells. Adding one key point on each edge and connecting them could generate a road map. This method is helpful for generating a simple path network; however, it does not create enough nodes in regular-shaped spaces.

\subsection{ArcGIS as a Platform}

ArcGIS has good support for network analysis; however, it is difficult to generate the path without the network layer. In ArcGIS, there is no tool to generate the mesh network directly with a flat 2D plane as the input. However, it does provide a tool to 
generate a fishnet for a plane of a given size. By adapting this tool with other 3D analyst tools, the fishnet has the potential to be used as a grid graph for referencing. It is therefore possible to use a mesh as an approach for path generation in ArcGIS. At the same time, ArcGIS also provides tools for Constrained Delaunay Triangulation which is termed as Triangulated Irregular Network (TIN). It is possible to utilize points and lines as inputs to generate the network. The user can use TIN as a base network and remove the unnecessary edges after completion. However, it is an arduous task to draw the minimal distance between corners and walls in ArcGIS. The overall process could be difficult.

Dijkstra (1959) provided a shortest path algorithm for calculating the minimum distance from one point to another. In GIS software like ArcGIS, it has already embedded functions for calculate the shortest path in 2D space. In ArcGIS 10 and later versions, Esri provides a tool to calculate the shortest path between two locations in the network dataset with elevation factor. The tool is under ArcTutorlNetwork AnalystlWorkflow 3 DRouting. As long as a valid 3D network dataset is provided, this tool could generate the shortest route between any two nodes that are on the network and export the result to the graph as a 3D tube.

\subsection{Summary}

Research has shown multiple ways of generating the indoor path network by using buildings' geometric data. However, adapting these methods and utilizing them for BIMs in ArcGIS is a challenge. After analyzing three approaches and discussing possible ways to operate them in ArcGIS, the result indicated that the mesh and TIN approaches seemed to be the better choices. However, implement those methods in ArcGIS could be difficult. 
The mesh approach requires each cell edge to be stored separately; however, the fishnet tool in ArcGIS generates the network and stores the whole data as combined rows and columns. On the other hand, the TIN approach needs a modification to its original methodology in order to be used in complex environments. In addition, BIMs are 3D data; the final path network requires a way to connect different floor levels. Therefore, in order to operate the mesh and TIN methods in ArcGIS, these problems need solutions. 


\section{Chapter 3 - Systems Analysis and Design}

This chapter is a general discussion of the project's design. This project was researchbased; the outcomes were methods and workflows. Therefore, it was crucial to specify the functional and non-functional requirements. Section 3.1 iterates the main study problem. The functional and non-functional requirements are summaries in Section 3.2. Section 3.3 reviews the design part of the system and includes the workflow of the whole system and flowcharts of each process. Section 3.4 discusses the plan for this project, followed by a summary in Section 3.5.

\subsection{Problem Statement}

Generating usable path network dataset for 3D architectural plans is difficult in current GIS and architecture software. In most cases, a path network dataset is required before performing any kind of network analysis. This is not usually a concern for outdoor GIS analysis since road data already carry basic information about the paths. For indoor environments, 3D architecture plans such as BIMs do not have the path feature embedded as a layer. Thus, the study problem is how to integrate BIM with GIS software to support indoor navigation.

\subsection{Requirements Analysis}

It was important to clarify the project requirements for system design and plan. Functional and non-functional requirements together directed the project and described the final output. 


\subsubsection{Functional Requirements}

Functional requirements define the inputs, behavior, and outputs of a system; a list of functional requirements for this project can be found in Table 1. A geodatabase was required for holding all the imported data from IFC. By running the designed models in ArcGIS, a 3D network dataset was generated for a building. After selecting two locations on the network and run the routing tool, the shortest path to the map is exported.

Table 1. Functional Requirements.

\begin{tabular}{|l|l|}
\hline \multicolumn{1}{|c|}{ Requirements } & \multicolumn{1}{c|}{ Description } \\
\hline Geodatabase & $\begin{array}{l}\text { Geodatabases shall store the data from IFC } \\
\text { files and hold intermediate data. The } \\
\text { geometrical components in IFC will be } \\
\text { transferred into feature classes in } \\
\text { geodatabases. }\end{array}$ \\
\hline $\begin{array}{l}\text { Path network generation method / } \\
\text { Workflow }\end{array}$ & $\begin{array}{l}\text { The method shall allow users to follow as a } \\
\text { guideline for path generation. By taking BIM } \\
\text { as the input, the method shall produce a } \\
\text { usable network dataset as an output. }\end{array}$ \\
\hline Routing Tool & $\begin{array}{l}\text { The routing tool shall take a network dataset } \\
\text { as an input, and calculate the shortest path } \\
\text { between two locations by giving two } \\
\text { locations as inputs. The routing result shall be } \\
\text { visually recognizable to users. }\end{array}$ \\
\hline
\end{tabular}

\subsubsection{Non-Functional Requirements}

The non-functional requirements included the description for method performance and system requirements for software; a list of non-functional requirements for this project can be found in Table 2 . 
Table 2. Non-functional Requirements.

\begin{tabular}{|l|l|}
\hline \multicolumn{1}{|c|}{ Requirements } & \multicolumn{1}{c|}{ Description } \\
\hline Data Interoperability & $\begin{array}{l}\text { IFC data was imported to ArcGIS, } \\
\text { the result shall be viewable on } \\
\text { different platform. }\end{array}$ \\
\hline Adaptability & $\begin{array}{l}\text { The methods shall be adaptable for } \\
\text { different buildings. }\end{array}$ \\
\hline 3D Visualization of Results & $\begin{array}{l}\text { The results shall be viewed in 3D in } \\
\text { ArcScene. }\end{array}$ \\
\hline
\end{tabular}

One important non-functional requirement was $3 \mathrm{D}$ visualization. It was not

required by the client, but was added to make the routing result be more appealing.

Another requirement is data interoperability, the final routing result shall be viewable on different platforms like Google map and Adobe 3D viewer, not only in ArcScene. The most important part was the adaptability of the methods, the methods shall be adaptable for different buildings ideally; however, adjustments are expected as applied to different buildings.

\subsection{System Design}

The general system design of this project is shown in Figure 3-1 which included four parts. The main steps included importing BIM to ArcGIS, generating a network dataset for the building, and running the routing algorithm to calculate the shortest path between two locations. Among these component, the critical component is path generation which is based on developing a usable 3D network dataset. 


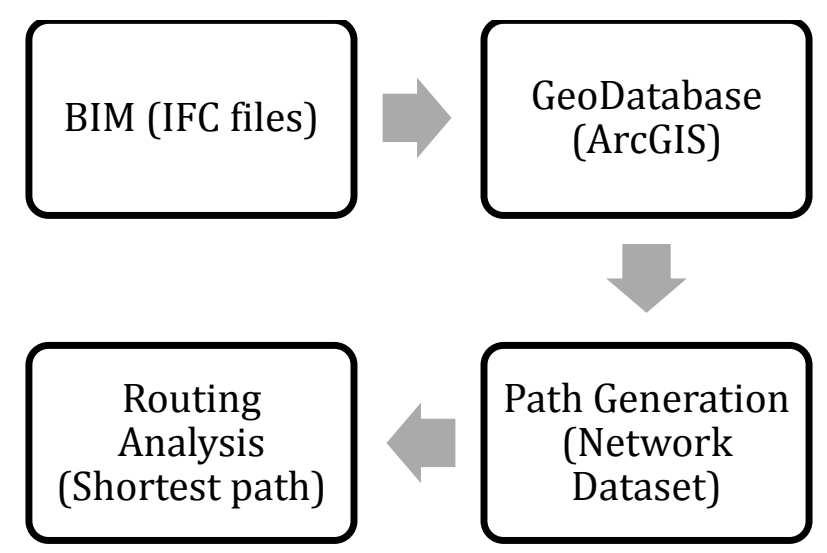

Figure 3-1: Project System Design

In this project, two approaches were proposed in path generation step: mesh approach and TIN approach. Section 3.3.1 and Section 3.3.2 present these two approaches. Section 3.3.3 gives a proof on the completeness of the TIN approach, explains in detail how to solve the Z-shaped hallway problem which was mentioned in Section 2.2 of Chapter 2.

\subsubsection{Mesh Approach}

The first path generation method is a mesh-based approach. A fishnet was generated for each floor by using the fishnet tool. Stair points were used to connect these networks and in the end, merging all the critical links to create the final network for the building. Fishnet was the main tool used to create the mesh network. The detailed workflow is available in Appendix A.

\subsubsection{TIN Approach}

An alternative network design was using the TIN model. Similar to the mesh method, stair points were used to connect the networks to a 3D network for the building after generating a usable path network for each floor. Two TIN networks were generated using this approach. The first applied the room vertices as nodes and the walls as critical edges 
to split the floor space. The second used the center points of the first TIN triangles, the center points of the first TIN edges that are not on the walls, and door points as nodes to create the base path network for the floor. Finally, the base path network became a serviceable network after removing the intersection edges with walls. Appendix B is the detailed workflow graph for this method.

\subsubsection{Proof for Completeness}

In order to solve the narrow Z-shaped hallway problem in the TIN approach, the center points of the first TIN edges that were not on the walls were included as part of the nodes in the final network. This section gives a proof on why these center points should be part of the node set.

Proof: $e_{n}$ is required for discribing any movements on $S$ without using $w_{n}$ (points on the wall)

Let $A$ be the floor plane, $C$ represents the set of all points on the plane.

Let $W$ be the set of points on the wall and $S$ be the set of points on the open space, then, $C=W \cup S$. After forming the 1 st TIN,

Let $T$ be the set contains all the points in the TIN triangles, Let $E$ be the set contains all the points on the TIN edges but not in $W$. then, $T \cup E=S$

Let $t_{n} \in T, e_{n} \in E, w_{n} \in W, n \in I, I=\{1,2,3, \ldots\}$

Then, without using $w_{n}$, traveling on $S$ is the movement from $t_{i}$ to $t_{j}$, or the movement from $t_{n}$ to $e_{n}$, or the movement from $e_{i}$ to $e_{j}, i \neq j$

Case $1 . t_{i}$ and $t_{j}$ are in the same triangle

Case $2 . t_{i}$ and $t_{j}$ are in different triangles 
So, after abstract each triangles as one point, Case 1 no longer exist.

$t_{i}$ and $t_{j}$ snap to the center points of the triangles.

Considering Case 2 , since $t_{i}$ and $t_{j}$ are srrounded by $e_{n}$,

$e_{n}$ has to participate in the traveling process.

The movement from $t_{n}$ to $e_{n}$ and

the movement from $e_{i}$ to $e_{j}$ each has at least one $e_{n}$.

Therefore, $e_{n}$ is required for discribing any movements on $S$ without using $w_{n}$.

In the end, after utilizing abstract edges as their center points, $e_{n}$ snaps to the centers, which then become the critical nodes. Therefore, it needs both $t_{n}$ and $e_{n}$ to simulates any movement on the 2D plane. Adding $e_{n}$ to the TIN approach solves the narrow Z-shaped hallway problem.

\subsection{Project Plan}

The original project plan consisted of two parts: integrating the BIM data with ArcGIS and use the imported data to perform routing analysis. The data were received in IFC format; the plan procedure began with investigative research on BIM and IFC with discovering the indoor navigation methods in ArcGIS as the primary task. In reality, after studying IFC file definition and path generation methods in computational geometry, the focus of the project changed to employing the geometric approach to build up a network dataset for BIM and using it as a base for routing analysis. ArcMap was used as the main platform for operation at the beginning. However, after investigating 3D analyst tools and multipatch feature classes, the working environment was moved to ArcScene and "3D visualization" was added as a criterion for the routing result. 


\subsection{Summary}

This chapter addressed the project requirements and provided an overview of system design. By using BIM as the input, this project aimed to provide solutions for path generation in ArcGIS. The functional requirements were as follows: finding automatic ways achieve the goal in ArcGIS (which required the process of importing BIM data), running algorithms, and performing routing analysis. The general workflow for this project was straightforward; the method design and operation were the challenging parts. Mesh and TIN were two approaches designed in ArcGIS. There are detailed descriptions of these processes in Chapter 5. 



\section{Chapter 4 - Data Import and Preparation}

The BIM data used in this project came in IFC format; therefore, in order to work with BIM data in ArcGIS, file transfer was required. A file geodatabase was used in this project since each dataset could be held as a file and scaled up to a terabyte in size. After

data transfer, it was necessary to scrub the data before running any algorithms. Data preparation was also an important step in this project. The path generation process was in 2D, so it was essential that the 3D multipatch features be abstracted as points or polylines before they were used in implementation. The following sections describe the steps of the data preparation.

\subsection{Data Source and IFC Data Model}

The BIM data provided by the client came in IFC format. These data could only be used for research purposes and this project used one building model as an example to show the path generation methods.

IFC formatted data have standard structures in holding data. The Data Interoperability extension in ArcGIS provides the ability to import BIM to ArcGIS (from IFC to a file geodatabase). However, the automatic transaction process does not inherit all the information from IFC. In order to customize the transformation process, the user can use the FME reader gallery and Spatial ETL tool. The IFC architectural model contains two kind of information: geometric and semantic. The geometric branch is the 3D representation of the building components and the semantic branch explains the relationships between these components. For this project, the automatic transaction process was used to provide data for path generation. Its general purpose was to simplify 
the process while still maintaining the critical components like the geometric properties of building parts (walls, doors etc.) and their width and height information.

\subsection{BIM to Geodatabase}

In order to operate the data transfer process in ArcGIS, an ArcGIS Advanced (ArcInfor) license and a Data Interoperability extension were required. Figure 4-1 shows the visualized features that viewed in ArcScene. The Quick Import tool from Data Interoperability extension was used with default settings. A file geodatabase is created for holding all the imported data which holds two types of data: tables and 3D feature classes. Tables contain the relationships for objects, and 3D feature classes are the 3D representation of building components.

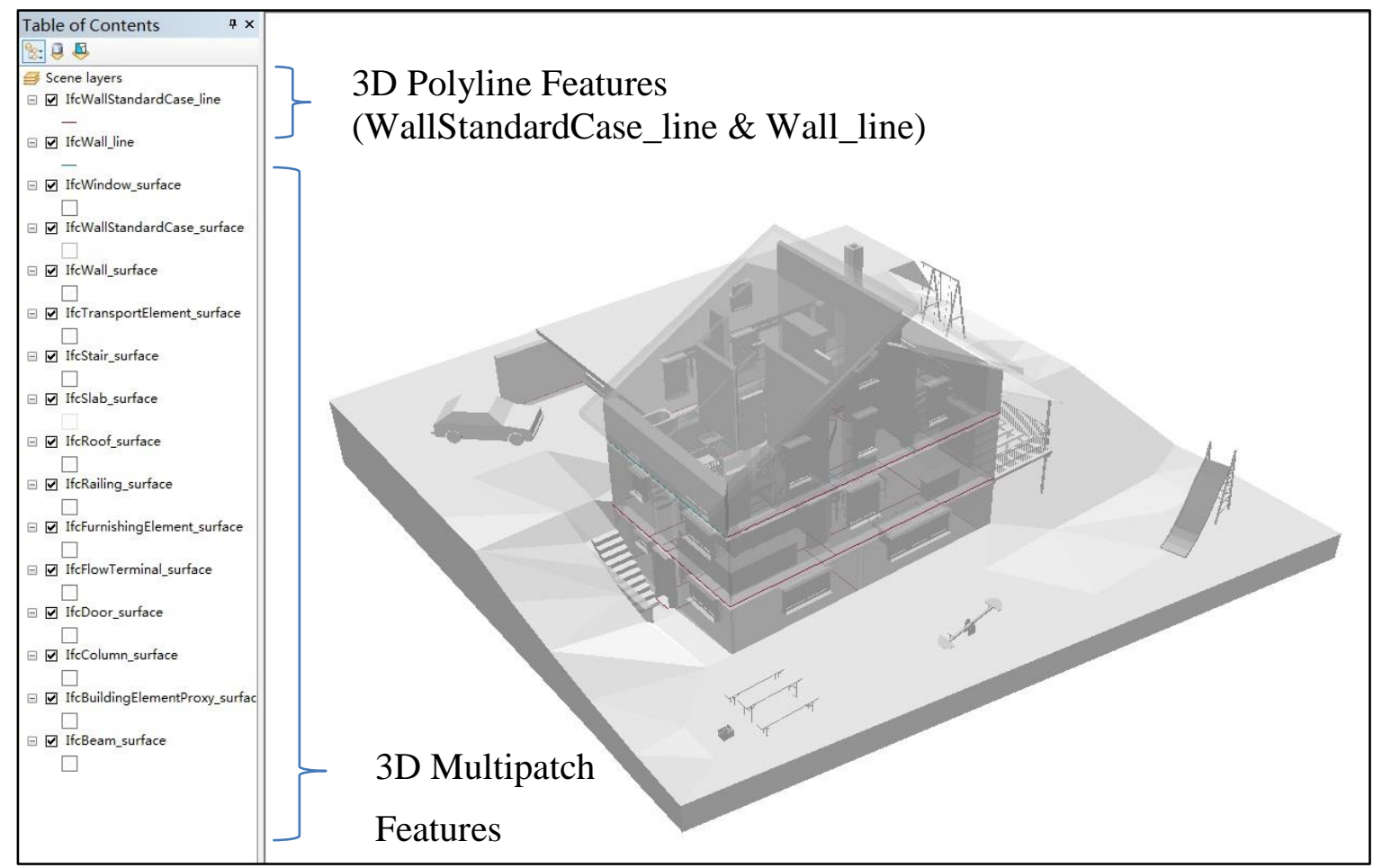

\section{Figure 4-1: BIM in ArcScene}




\subsection{Data Scrubbing}

Table 3 lists the required feature classes from the geodatabase, along with the data that work as references. Both mesh and TIN approach required data scrubbing before further process can be performed.

\section{Table 3. Data Description}

\begin{tabular}{|l|l|}
\hline \multicolumn{1}{|c|}{ Required Data } & \multicolumn{1}{c|}{ Reference data } \\
\hline IfcDoor_surface & IfcRoof_surface \\
\hline IfcWall_suface & IfcSlab_surface \\
\hline IfcWall_line & IfcWindow_surface \\
\hline IfcWallStandardCase_surface & IfcBuildingElementProxy_surface \\
\hline IfcWallStandardCase_line & IfcSpace_surface (optional) \\
\hline IfcStair_surface & \\
\hline
\end{tabular}

IfcWallStandardCase_line and IfcWall_line are two 3D polyline feature classes that represent the footprints of walls on the floors. These features carry the geometric information about the room scale as well as the locations of internal walls and pillars. IfcWall, IfcDoor, IfcStair, IfcWindow, IfcRoof, and IfcSlab belong to IfcBuildingElement, and within them, IfcWall contains IfcWallStandardCase.

ArcGIS provides editing tools for simple fix if there exist any space connectivity errors. Those errors are not really errors in architecture sense, but are considered as errors in space connectivity sense. For example, Figure 4-2 demonstrates the process of error fixing to this concern. Figure 4-2 (a) shows the gaps and between walls after walls were merged together into one polyline feature "Wall_line". The Wall_line feature class needs to be checked to ensure the external walls and boundary walls are connected to each other without any gaps. If a gap is found, the Extend Line tool could be used to extend the lines and fill the gap. (b) The fixed floor plan will then be useful in the implementation part. 




(a)

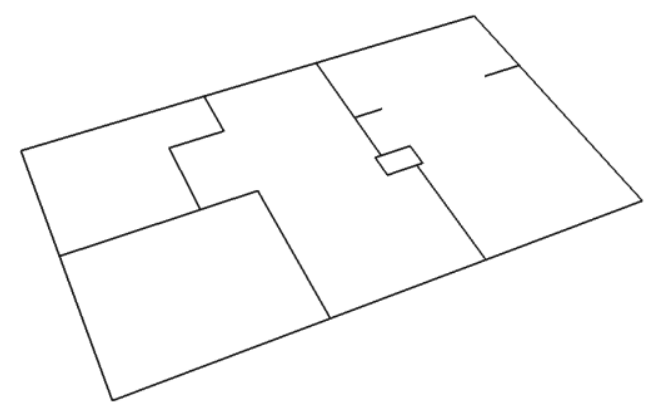

(b)

\section{Figure 4-2: Editing Wall Features}

\subsection{Data Preparation}

Further data editing and preparation were required before implementation. BIM data are an excellent source for construction and management, but in order to use them efficiently for 2D path generation, critical components like doors and stairs have to be transferred into spatial points in advance. At the same time, these new spatial points should inherit attributes from their original multipath features. The workflow of this design can be found in Appendix C. 


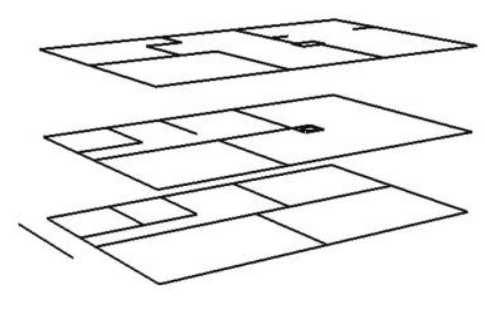

(a)

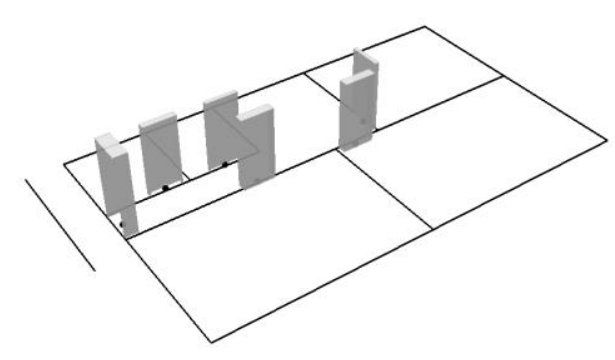

(c)

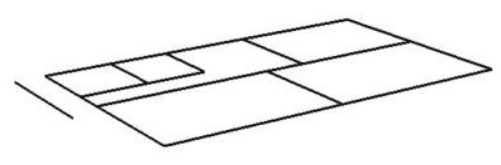

(b)

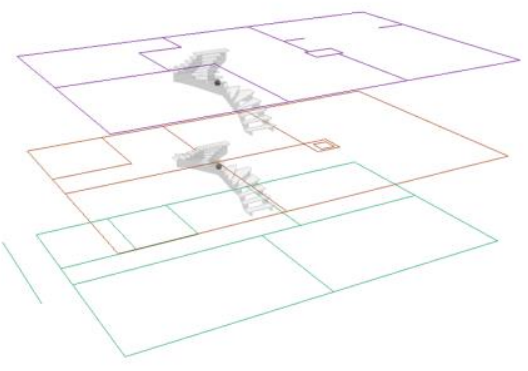

(d)

\section{Figure 4-3: Data Preparation}

Figure 4-3 displays some important parts in the data preparation process. Figure 4-3 (a) and (b) together show the output of separating floor plans according to their height, the elevation field was used as the height reference. The doors were abstracted as points on each floor which were illustrated in Figure 4-3 (c). Staircases were also abstracted as points in Figure 4-3 (d) and the elevation value was determined by their center points' height. The stair points were served as the jump points between floors. Although it is not a perfect abstraction method, it can function as a compromise for connecting floors.

\subsection{Summary}

In order to work with BIM data, it is necessary to import them into file geodatabases. Since both path generation methods are geometric approaches, 3D polyline feature classes and multipatch feature classes are the main inputs for implementation. After the 
data cleaning process is complete, the user can abstract some critical building components from the multipatch feature into points for 2D path generation. 


\section{Chapter 5 - Implementation}

Mesh and TIN methods were proposed in this project. As mentioned in Chapter 2, the primary challenges faced in implementation were adjusting the path generation methods and operating them in ArcGIS. This chapter covers in detail the operation processes for both methods, which are visualized in ArcScene. Section 5.1 and 5.2 introduce the basic theories behind these approaches, followed by the workflow used to implement them. The chapter ends with a summary.

\subsection{Mesh Approach}

The mesh method requires the use of a standard-sized grid graph as the referencing network for a floor and subsequently map the movement on a $2 \mathrm{D}$ plane to the movement along grid edges. A fishnet created covering the floor serve as the referencing network for path generation. Mesh is therefore the path generation. The fishnet tool in ArcGIS was used to create a base mesh network for each floor; after removing the intersecting edges with walls, the fishnet became an effective path network for the corresponding floor.

The following paragraphs systematically explain how to operate this mesh approach in ArcScene. 


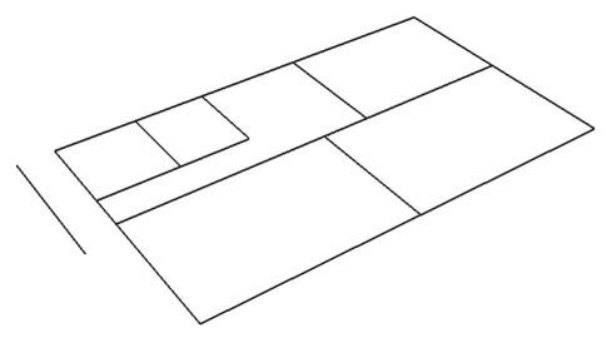

(a)

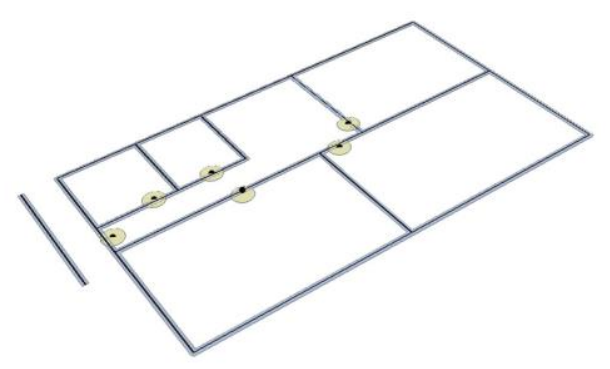

(c)

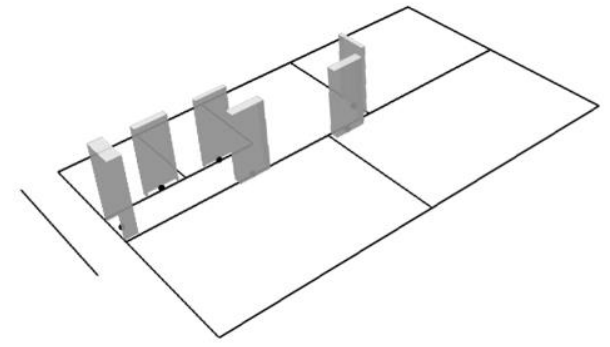

(b)

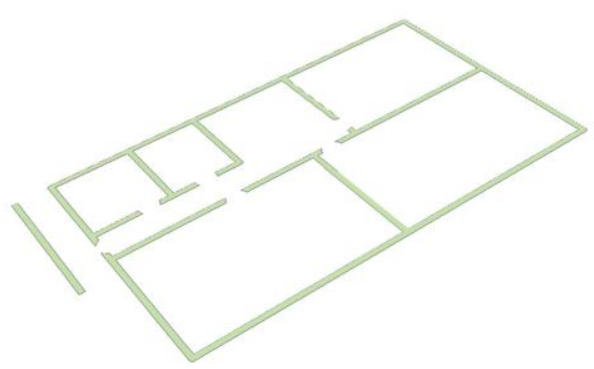

(d)

\section{Figure 5-1: Preparing a Floor Plan}

Figure 5-1 and Figure 5-2 together demonstrate the process of performing the mesh approach. For each separate floor plan, Figure 5-1 (a), as an example, shows that the integrated wall line feature class was used as the start platform. The doors were abstracted into points in the data preparation step as shown in Figure 5-1 (b). Figure 5-1 (c) displays the buffers around walls and doors created by taking the door width and wall width as the referencing value. The wall buffer was then used as the input feature and door buffer as the erase feature to erase the doors from the wall. The result is displayed in Figure 5-1 (d), a polygon feature class which represents the cleaned floor plan including rooms and doors. 


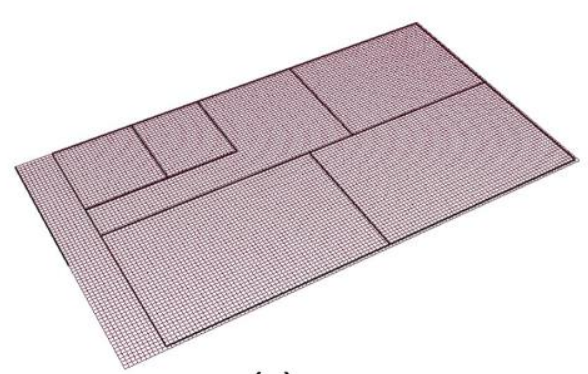

(a)

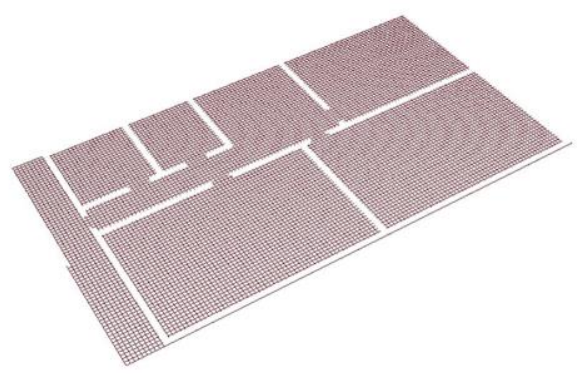

(c)



(b)



(d)

\section{Figure 5-2: Creating a Fishnet Template for a Floor Plan}

The Create Fishnet tool was then utilized to generate a fishnet template for each floor as shown in Figure 5-2 (a). The floor plan's layout was used to ensure the fishnet template covers the floor surface entirely. The width and height of the cells in the fishnet were set to $0.1 \mathrm{~m}$ in this case, and this value could be reduced or raised to either increase or decrease the precision of the fishnet. In addition, the cell's width and height have to be smaller than half of the door width value. Otherwise it has the risk of producing a closed room. In Figure 5-2 (b), the cleaned floor plan from Figure 5-1 (d) was placed to overlay with the fishnet. The fishnet was then set as the input feature and the floor plan as the erase feature to erase the walls from the fishnet. The output, as showed in Figure 5-2 (c), is the final mesh network for the floor. The nodes of this network were created by 
applying the Feature Vertices to Points tool on it. Figure 5-2 (d) demonstrates the output of adding the connection to the above stair case point to the network.

Figure 5-3 is the final network for the entire building. A usable network dataset could be created by importing the merged mesh network to the feature dataset, specifying the geometry length as the cost field, and employing $\mathrm{Z}$ coordinate values from geometry as the elevation model.

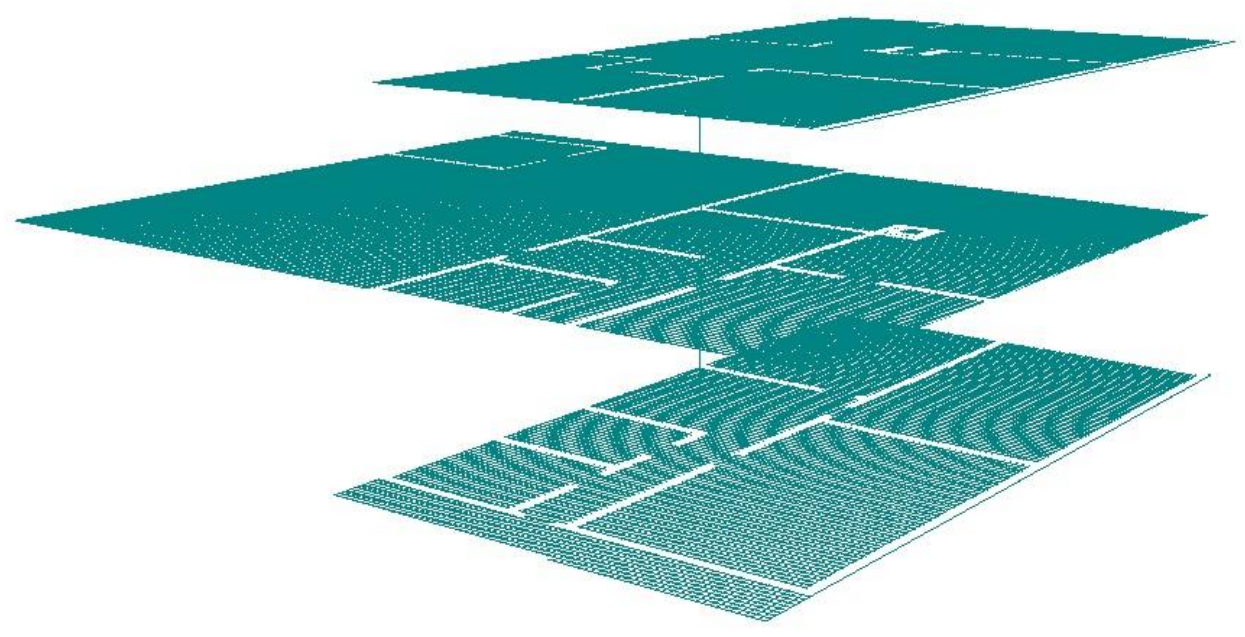

Figure 5-3: Mesh Network for Multiple Floors

This final network dataset served as the basis for indoor routing. 


\subsection{TIN Approach}

The second approach involved mapping the movement on a $2 \mathrm{D}$ plane to the movement along TIN edges using TIN graphs. Walls and room vertices were used to generate the first TIN network. It was then necessary to generate the center points of TIN triangles and the center points of TIN edges that did not fall along the wall line as walls are the barriers for movement. By using these points, together with the door points as nodes to create a second TIN network, this network could be used as the path network after removing the edges that were intersecting with the walls.

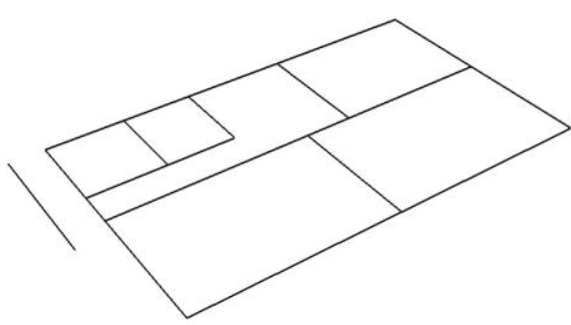

(a)

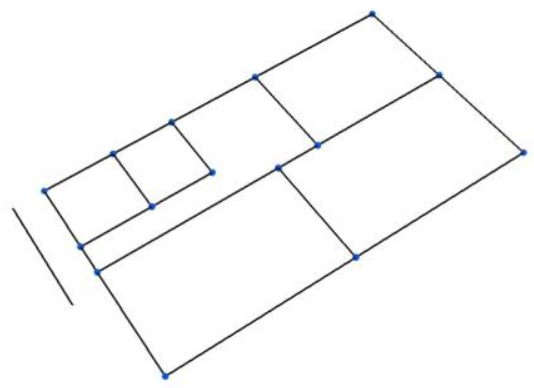

(c)

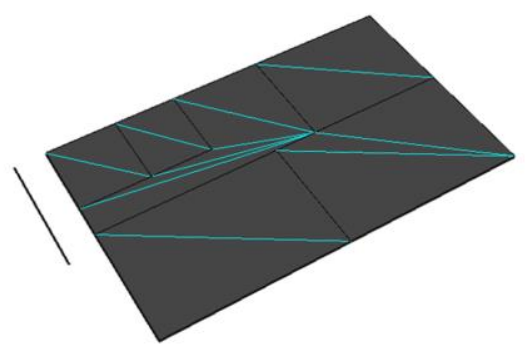

(b)

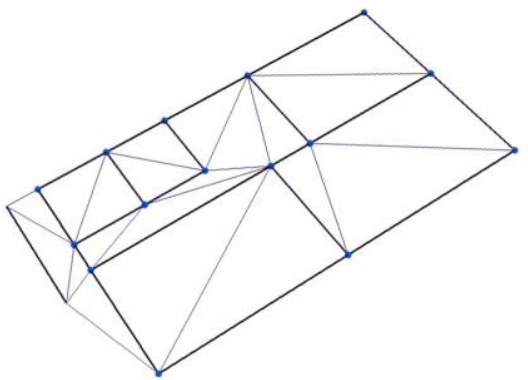

(d)

\section{Figure 5-4: TIN Part 1}

Figure 5-4 and Figure 5-5 present the TIN approach. The TIN method started with the same wall feature as shown in Figure 5-4 (a). Figure 5-4 (b) displays the identification 
of polygons on the floor plan by using Feature to Polygon tool. This tool generated a polygon for each room and hallway. The vertices of the polygons were then extracted (Figure 5-4 (c)). Using the walls as hard lines (critical edges) and vertices from Figure 54 (c) as mass points (nodes), the first TIN network was created as illustrated in Figure 5-4 (d). The TIN Triangle and TIN Edge tools were utilized to get TIN triangles and edges as separate feature classes.



(a)

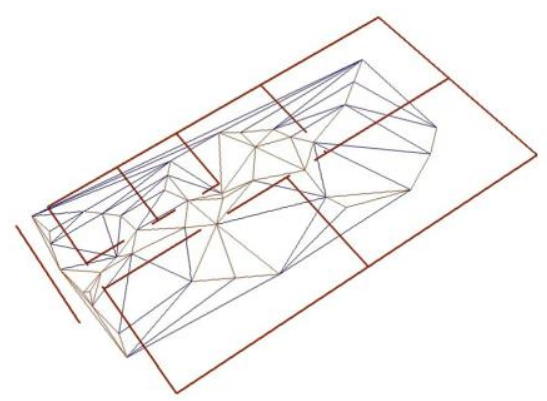

(c)



(b)

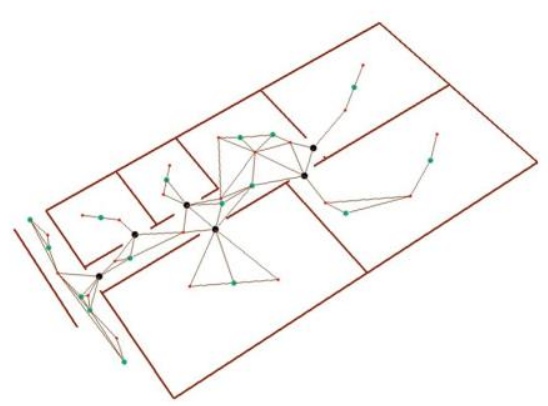

(d)

\section{Figure 5-5: TIN Part 2}

Figure 5-5 (a) shows the inside center of the TIN triangles and TIN edge features which were not intersect with the wall lines. Since doors are part of path, door should be included to the network. Thus, the walls were adjusted to leave the door open (Figure 55(b)). The door points and the center points from Figure 5-5 (a) were merged and used as mass points (nodes) to create the floor's second TIN network as demonstrated in Figure 
5-5 (c). After removing the edges of the TIN network that intersect with walls, the final network for this floor was complete as displayed in Figure 5-5 (d).



Figure 5-6: TIN Stair Connection

Figure 5-6 exhibits the connection of the floor network and the star cases above it. The stair case point is only used as a referencing point, the actually connection between floors may not passes it. All the path networks and stair connections in the building were merged to create the path network of this building as shown in Figure 5-7.



Figure 5-7: TIN Network for Multiple Floors 


\subsection{Summary}

This project employed the mesh and TIN approaches for path generation in ArcGIS,

Both of these proposed methods generated the path network successfully. Using ArcGIS

to perform path generation is not an easy task - modifying the results generated by $2 \mathrm{D}$

analysis and utilizing them for the $3 \mathrm{D}$ process is the key. 


\section{Chapter 6 - Results and Analysis}

This chapter focuses on the routing results of this project, which were calculated using network analysis tools. A detailed description of the routing tool can be found in Chapter 2. The final outputs of this project are methods and workflows that explain the path generation processes in ArcGIS, and these can be used to generate a path network for each floor.

The first section includes graphs of the routing results for both the mesh and TIN networks, followed by a comparative analysis in Section 6.2. Section 6.3 provides the result export options in ArcGIS.

\subsection{Routing Result}

Once the network datasets were created for the building, the 3D routing tool provided in ArcTutor/3D routing could produce the routing result by taking the network dataset as the input and choose two locations on the network. The shortest path between these two locations will be added to the graph as a 3D tube. Figure 6-1 (a) shows the routing result by taking mesh network dataset as the input, (b) presents the result using TIN network dataset as the input.



(a)



(b)

Figure 6-1: Routing Result 
Both approaches served the purpose of indoor routing well. The general processing time of TIN was shorter than Mesh since TIN contains less nodes and edges than Mesh. However, stair connections tend to compromise floor linkage. In this project, stairs cases were abstracted into points which are floating between floor levels. The stair connections were simply the connections from the stair points to the nearest point on the floor above it and below it. So, current solution is more like an elevator in functionality sense. In order to have better approach to the real world and attain usable results for multi-story buildings, further adjustments were needed for generating correct geometric connections between floor planes and more complex solution needs to be proposed. An ideal solution would be finding the staircase rooms on each floor and connection them in space, and this action would require the integration of more sematic information.

\subsection{Comparison between Mesh and TIN Approaches}

Each approach generates a final path network that is usable for each floor. The comparison of the two methods is in Table 4.

Table 4. Mesh and TIN Comparison

\begin{tabular}{|l|l|l|}
\hline \multicolumn{1}{|c|}{ Methods } & \multicolumn{1}{c|}{ Advantages } & \multicolumn{1}{c|}{ Disadvantages } \\
\hline Mesh approach & $\begin{array}{l}\text { The output path network is } \\
\text { very precise and it has } \\
\text { equal coverage density for } \\
\text { each room. }\end{array}$ & $\begin{array}{l}\text { It consumes more storage } \\
\text { space. }\end{array}$ \\
\hline TIN approach & $\begin{array}{l}\text { It is an efficient and easy } \\
\text { way of generating the path, } \\
\text { and it requires less storage } \\
\text { space. }\end{array}$ & $\begin{array}{l}\text { It does not have equal } \\
\text { coverage density in each } \\
\text { room. }\end{array}$ \\
\hline
\end{tabular}


Although the mesh method consumes more storage space than the TIN network, it generates a more precise path network for each floor in general. The subdivisions used in this approach are regular grids, thus the accuracy level of the mesh network is adjustable by increasing or decreasing the grid size. In ArcGIS, the fishnet tool provides the foundation for the mesh network. This project used it as its path-referencing network. The final network dataset not only can be used for visualization purposes but also for routing analysis.

As a modified method of the Constrained Delaunay Triangulation approach, the TIN approach uses the center points of TIN edges as extra mass points to participate in the final TIN network generation.

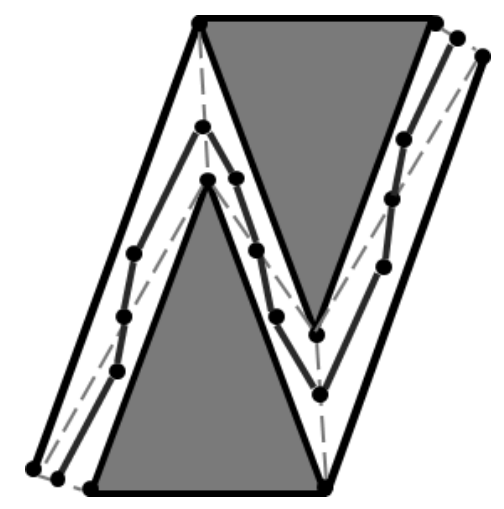

Figure 6-2: Solved Z-Hall Way Problem

Since TIN edges work as boundaries between subdivisions (TIN triangles), the user can abstract them into points and include them as part of the nodes meant to fix the topological links between adjacent corner subdivisions. Figure 6-2 presents the modified path by taking the center points of edges into nodes, it solves the Z-hall way problem mentioned in Chapter 2. It is also possible to use the center points of TIN edges alone as mass points to generate the path network. 
The TIN approach generates the path network by only taking critical points as nodes; therefore, the overall generation process is swift. However, a TIN network is not able to provide point-to-point navigation randomly in space, densities of nodes are not equally across the pace. One possible alternative method for improvement would be generate multi-depth TIN networks, by using the center points of current subdivisions as extra nodes to create next TIN network, repeating the process several times to increase the density of nodes.

\subsection{Export Solutions}

After receiving the routing results, Quick Export in the Data Interoperability extension can export the solution to any 3D file format that is supported by the FME writer Gallery embedded in ArcGIS. Most commonly used 3D file formats (e.g. Adobe 3D, AutoCAD, Google Earth KML, and CityGML) are supported. The network dataset itself could also be exported to these formats.

\subsection{Summary}

Both the mesh and TIN approaches generate usable path networks for each floor. In this project, stairs are imported as detailed multipatch features using the Quick Import tool in the Data Interoperability extension. Their attributes do not have the connection information that can be used to create geometric links. Therefore, as a compromise for linkage, this project abstracted stairs as points and used them to fashion connections between floors. The final network datasets are functional in ArcGIS, which makes it valuable for BIM integration in regards to operating spatial selections and performing network analysis. The result of this project is a sound starting point for the use of ArcGIS to generate visible indoor paths for BIM. 


\section{Chapter 7 - Conclusions and Future Work}

This chapter summarizes the project's procedures, describes how the initial goals were archived, explores fields for future work, and investigates potential application areas.

\subsection{Conclusions}

The goal of this paper was finding automatic and executable ways to generate network data-sets for BIMs in ArcGIS. The general idea is utilizing the 3D architecture plans for indoor navigation and use ArcGIS as a platform to both performs the generating process and routing analysis. Two methods were proposed to complete this goal. The mesh approach provided a precise path network for each floor. In comparison, TIN required less storage space and processing time but only utilized a limited number of nodes in its constructing network, and the nodes did not cover the space in equal density. Once the network dataset was created, the 3D Routing tool in ArcGIS generated the desired path by taking locations as inputs. ArcGIS also provides tools in Data Interoperability to export the solutions to many commonly used 3D formats.

\subsection{Future Work}

This project attempted to build a starting point for the use of ArcGIS as a platform in path generation and routing analysis for BIMs. The result shows the possibility of applying it to real objects and points to the value of further investigation. Presently, the solutions proposed by this project are usable for path generation on each building floor, but stairs present a problem in floor linkage. Hence, improving the data import method and finding other methods to create accurate connections between floor levels are suitable future endeavors. In addition, merging the semantic information with visualized results would 
help routing analysis and extend its potential usages. Another field of expansion could be combining the indoor navigation system and outdoor GPS system together, creating a seamless connection within a point-to-point navigation system. At the moment, there is limited support for path generation in ArcGIS; however, with the development of indoor 3D analysis in the GIS world, the integration between BIM and GIS is gaining in strength. 


\section{Works Cited}

ArcGIS.10.Help. (2012). Analysis on 3D network datasets. Retrieved from ArcGIS Resource Center: http://help.arcgis.com/en/arcgisdesktop/10.0/help/index.html\#//00470000005q000 000

Autodesk. (2012). BIM Overview. Retrieved from autodesk website: http://www.autodesk.com/solutions/bim/overview

Benner, J., \& Leinemann, K. (2005). Flexible Generation of Semantic 3 D Building Models. Proc of the 1st Intern. Workshop on Next Generation 3D City Models.

Berlo, L. v., \& Laat, R. d. (2010, November 3-4). Integration of BIM and GIS: The development of the CityGML GeoBIM extension. 5th International 3D GeoInfo Conference. Berlin, Germany.

Borrmann, A. (2010, November 3). From GIS to BIM and Back Again - A Spatial Query Language For 3D Building Models and 3D City Models. International Archives of the Photogrammetry, Remote Sensing and Spatial Information Sciences, XXXVIII4/W15.

BuildingSmart. (2010, Oct 01). Retrieved from www.buildingsmart-tech.org: http://www.buildingsmart-tech.org/ifc/IFC2x4/rc2/html/schema/chapter-5.htm

Chew, P. L. (1987). Constrained Delaunay Triangulations. Proceedings of the Third Annual Symposium on Computational Geometry.

Dijkstra, E. W. (1959). A note on two problems in connexion with graphs. Numerische mathematik 1(1), 269-271. 
Dudas, P. M., Ghafourian, M., \& Karimi, H. A. (2009). ONALIN: Ontology and Algorithm for Indoor Routing. University of Pittsburgh, Geoinformatics Laboratory -School of Information Sciences. Tenth International Conference on Mobile Data Management. doi:10.1109/MDM.2009.123

El Meouche, R., Rezoug, M., \& Hijazi, I. (2013). Integrating and Managing BIM in GIS, Software Review. ISPRS 8th 3DGeoInfo Conference \& WG II/2 Workshop. Istanbul, Turkey.

El-Mekawy, M. (2010). Integrating BIM and GIS for 3D city modelling-The Case of IFC and CityGML. Royal Institute of Technology (KTH).

El-Mekawy, M., Östman, A., \& Hijazi, I. (2012, July 17). A Unified Building Model for 3D Urban GIS. ISPRS International Journal of Geo-Information, 1, pp. 120-145. doi:10.3390/ijgi1020120

El-Mekawy, M., Östman, A., \& Hijazi, I. (2012). An Evaluation of IFC-CityGML Unidirectional Conversion. (IJACSA) International Journal of Advanced Computer Science and Applications, 3(5).

GSA (2007). GSA BIM Guide Overview. Retrieved May 20, 2014, from: http://www.gsa.gov/portal/mediaId/226771/fileName/GSA_BIM_Guide_v0_60_S eries01_Overview_05_14_07.action

Hijazi, I., Ehlers, M., \& Zlatanova, S. (2010, November). BIM for Geo-analysis (BIM4GEOA): Set up of 3D Information System with Open source Software and Open Specification (OS). International Archives of the Photogrammetry, Remote Sensing and Spatial Information Sciences, XXXVIII-4/W15. 
Irizarry, J., \& Karan, E. P. (2012, August). Optimazing Location of Towercranes on Construction. Journal of Information Technology in Construction (ITcon), Vol. 17, pp. 351-366. Retrieved from http://www.itcon.org/2012/23

ISA Project Team. (2008). What is Building Information Modeling (BIM)?

Karola, A., Hitchcock, R., Chen, Q., Dajka, S., \& Hagström, K. (2002). BSPro COMServerinteroperability between software tools using industrial foundation classes. Energy and, 34(9), pp. 901-907.

Lamarche, F., \& Donikian, S. (2004). Crowd of Virtual Humans: a New Approach for Real Time Navigation in Complex and Structured Environments. IRISA, Campus de Beaulieu, F-35042 Rennes, FRANCE: Computer Graphics Forum. doi:10.1111/j.1467-8659.2004.00782.x

Lin, Y.-H., Liu, Y.-S., Gao, G., Han, X.-G., Lai, C.-Y., \& Gu, M. (2003). The IFC-based path planning for 3D indoor spaces. Advanced Engineering Informatics.

Liu, L. (2011). 3D indoor "door-to-door" navigation approach to support first responders in emergency response. Jaffalaan 9, 2628 BX Delft, the Netherlands: OTB Research Institute for Housing, Urban and Mobility Studies.

Mas, S., Reinhardt, W., \& Wang, F. (2006). Conception of a 3D Geodata Web Service for the Support of Indoor Navigation with GNSS. Innovations in 3D Geoinformation Science, pp. 307-316.

Monteiro, A. (2012). SIGABIM: a framework for BIM application. Istanbul, Turkey: IAHS World Congress.

Nagel, C., Becker, T., Kaden, R., Li, K.-J., Lee, J., \& Kolbe, T. H. (2010). Requirements and Space-Event Modeling. Open Geospatial Consortium. 
Rich, S., \& Davis, K. H. (2010). Geographic Information Systems (GIS) for Facility. IFMA Foundation.

Rueppel, U., \& Stuebbe, K. (2008, October). BIM-Based Indoor-Emergency-NavigationSystem for Complex Buildings. Tsinghua Science and Technology, 13(S1), pp. $362-367$.

Rüppel, U., Abolghasemzadeh, P., \& Stübbe, K. (2010). BIM-based immersive indoor graph networks for emergency situations in buildings. Retrieved from http://www.academia.edu/: http://www.academia.edu/2560918

Sharkawi, K., Ujang, M., \& Abdul-Rahman, A. (2008). 3D Navigation System for Virtual Reality Based on 3D Gaming Engine. The International Archives of the Photogrammetry, Remote Sensing and Spatial Information Sciences, XXXVII. Part B4.

Tulke, J., Nour, M., \& Beucke, K. (2008). A Dynamic Frameworkfor Construction Scheduling based on BIM using IFC. Chicago, USA: Proc. 17th Congress of IABSE.

Xu, W., Kruminaite, M., Onrust, B., Liu, H., Xiong, Q., \& Zlatanova, S. (2013, December). A 3D Model Based Indoor Navigation System For Hubei Provincial Museum. The International Archives of the Photogrammetry, Remote Sensing and Spatial Information Sciences, XL-4/W4. doi:10.5194/isprsarchives-XL-4-W4-512013

Zhang, X., Arayici, Y., Abbott, C., \& Aouad, G. (2009). Integrating BIM and GIS for large scale (building) asset management: a critical review. University of Salford. 


\section{Appendix A. Workflows-Mesh}

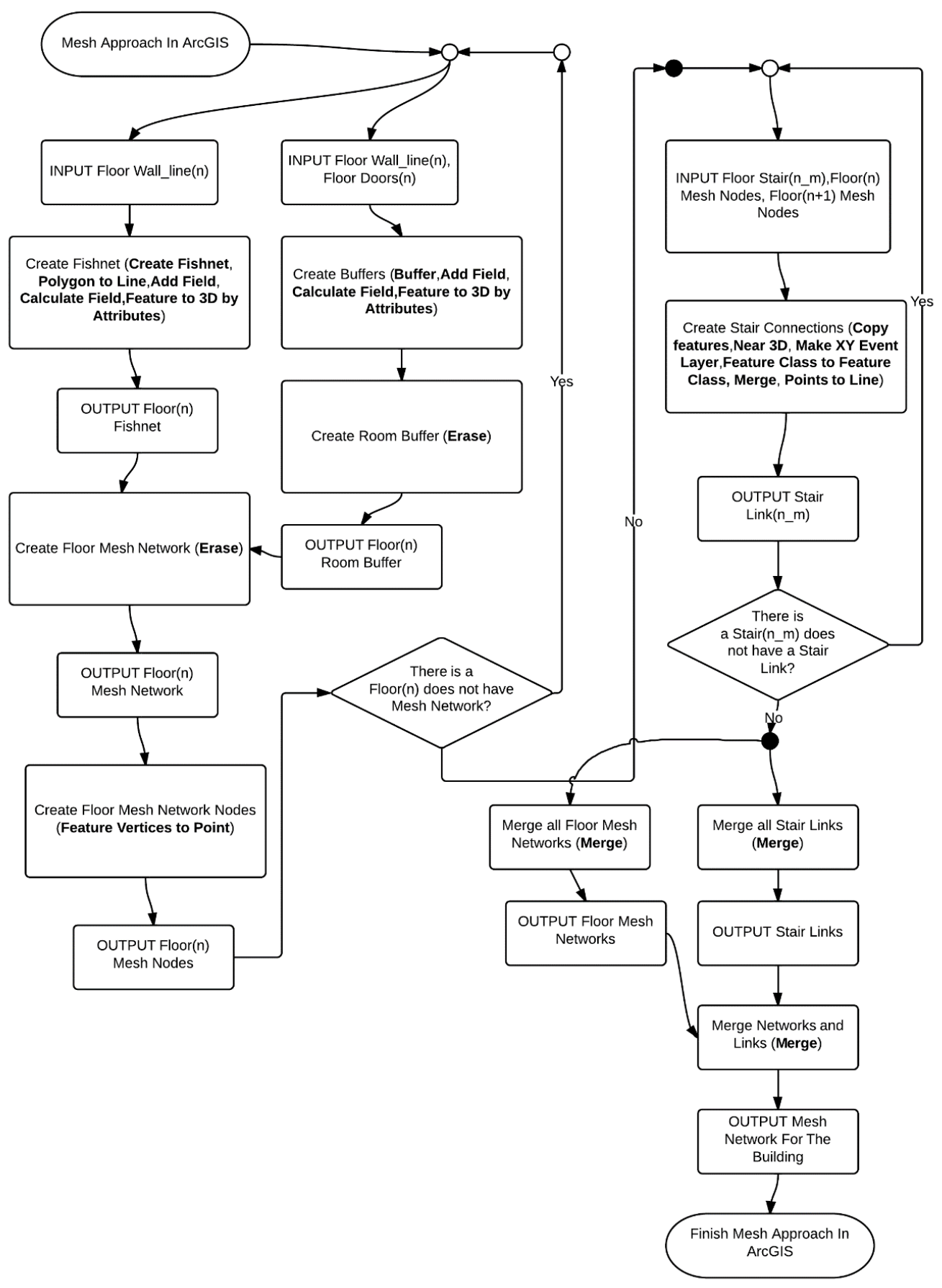




\section{Appendix B. Workflows-TIN}

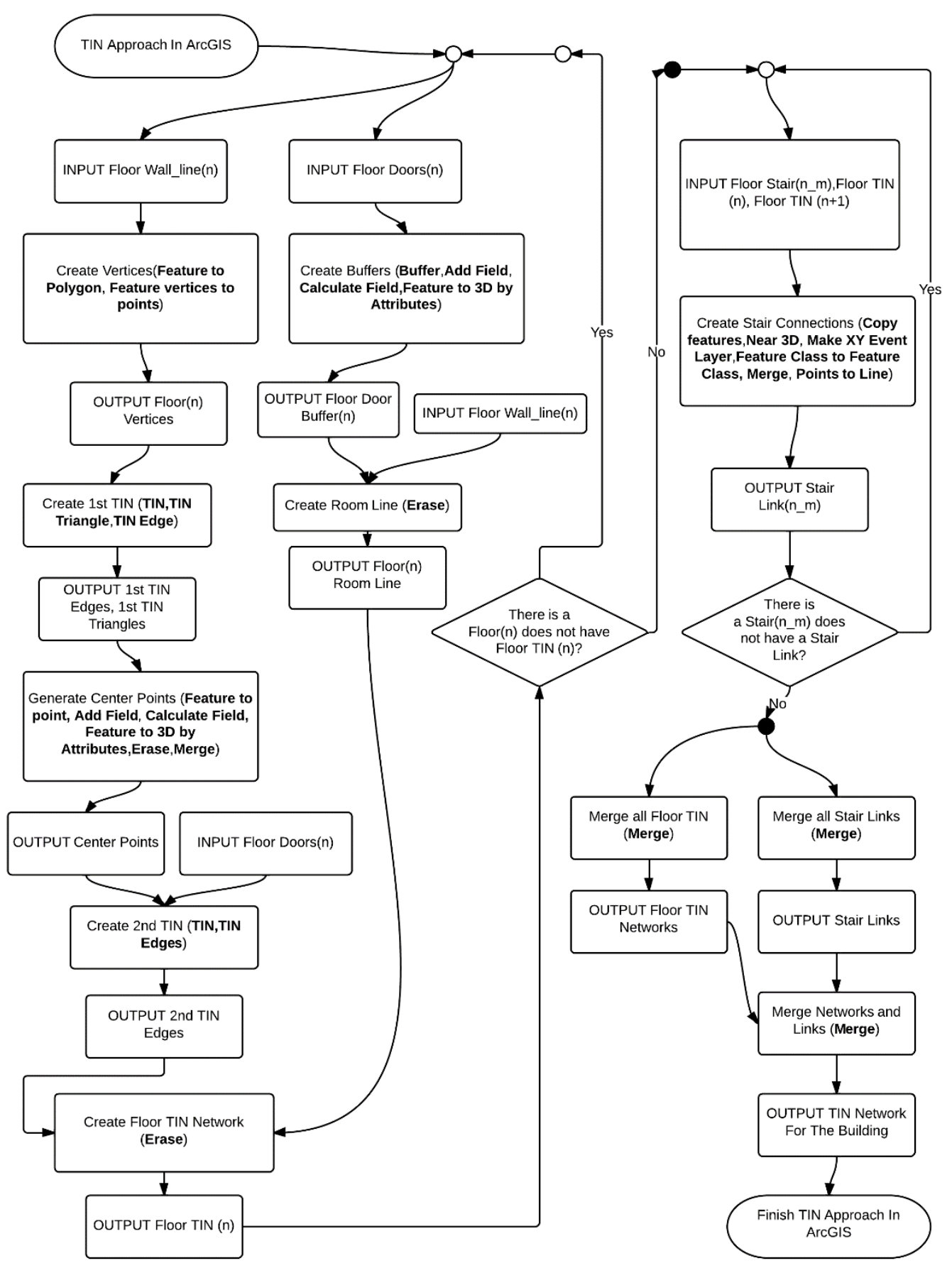




\section{Appendix C. Workflows-Data Preparation}



